\title{
Relationship between Patient-Dependent Parameters and Radiation Dose Rates Measured around Patients Undergoing PET/CT Imaging Using 18F-FDG
}

\author{
Khaled Soliman ${ }^{1 *}$, Saad Al Qahtani' ${ }^{2}$, Ahmed Alenezi ${ }^{3}$ \\ ${ }^{1}$ Medical Physics Department, Prince Sultan Military Medical City, Riyadh, Saudi Arabia \\ ${ }^{2}$ Radiodiagnostic and Medical Imaging Department, Prince Sultan Military Medical City, Riyadh, Saudi Arabia \\ ${ }^{3}$ Faculty of Applied Medical Sciences, King Saud University, Riyadh, Saudi Arabia \\ Email: *khaledsoliman61@gmail.com
}

How to cite this paper: Soliman, K., Al Qahtani, S. and Alenezi, A. (2018) Relationship between Patient-Dependent Parameters and Radiation Dose Rates Measured around Patients Undergoing PET/CT Imaging Using ${ }^{18} \mathrm{~F}$-FDG. International Journal of Medical Physics, Clinical Engineering and Radiation Oncology, 7, 403-413.

https://doi.org/10.4236/ijmpcero.2018.73033

Received: August 6, 2018

Accepted: August 27, 2018

Published: August 30, 2018

Copyright $\odot 2018$ by authors and Scientific Research Publishing Inc. This work is licensed under the Creative Commons Attribution International License (CC BY 4.0).

http://creativecommons.org/licenses/by/4.0/

\section{(c) (i) Open Access}

\begin{abstract}
Objectives: Patients undergoing ${ }^{18} \mathrm{~F}$-FDG PET/CT imaging are considered external radiation sources. Accurate dose rate estimates are important for conducting realistic risk assessments and performing dose reconstruction in cases of accidental exposures. The patient radiation self-attenuation factor is assumed to be a function of the patient's body size metrics, but we can use these metrics to predict the dose rate around the patients with accuracy. The objective of this work was first to measure the patient attenuation factor by performing direct dose rate measurements from patients undergoing PET/CT imaging studies using ${ }^{18} \mathrm{~F}$-FDG. The second objective was to study the possible correlation between the measured dose rate constant per unit activity from the patients and their body size metrics; five metrics were tested in this work. The last objective was to measure the patients' voiding factor. Methods: We have measured dose rates at one meter from 57 patients and noted the patient's height $(\mathrm{H})$, weight $(\mathrm{W})$ and calculated patient size metrics namely: Equivalent Cylindrical Diameter (ECD), Equivalent Spherical Diameter (ESD) and the Body Mass Index (BMI). Results: The measured average dose rate was $92.2 \pm 14 \mu \mathrm{Sv} \cdot \mathrm{h}^{-1} \cdot \mathrm{GBq}^{-1}$ measured at one meter. Therefore, the dose rate constant of $92 \mu \mathrm{Sv} \cdot \mathrm{h}^{-1} \cdot \mathrm{GBq}^{-1}$ proposed by the AAPM, TG-108 report is adequate for radiation protection purposes. There was no statistically significant correlation between the dose rate constant per unit activity and the patient body size metrics. We have measured a patient voiding factor of $0.89 \pm 0.06$ in comparison with 0.85 recommended by the AAPM. Conclusions: The presented data can be used by medical physicist working in nuclear medicine in formulating more accurate risk estimations resulting from radiation exposure from patients undergoing ${ }^{18} \mathrm{~F}-\mathrm{FDG}$ PET/CT imaging.
\end{abstract}




\section{Keywords}

FDG, Measured Dose Rate, Patient Voiding Factor, Patient Attenuation Factor, Patient Size

\section{Introduction}

The estimated dose rate at certain distance from a radioactive source depends on the dose rate constant, the source activity and the distance between the source and the measurement point. Once the radioactivity is incorporated into the patient, it will additionally depend on his body tissues attenuation properties.

Accurate dose rate estimates are important for radiation protection specialists conducting risk assessments and performing dose reconstruction in cases of accidental exposures. The American association of physicist in medicine (AAPM) in their report TG-108 shielding design for positron emitted tomography/computed tomography (PET/CT) imaging facilities recommends the use of a dose rate constant of $92 \mu \mathrm{Sv} / \mathrm{h} / \mathrm{GBq}$ for Fluorine-18 based compounds in situation where the patient is considered the source of radiation exposure [1]. The value proposed by the AAPM is used in the calculations of the facility shielding design, where significant cost savings are achieved by using optimized designs while eliminating the use of additional shielding materials thicknesses. In the same AAPM report, it is also suggested to use a patient voiding factor of 0.85 in order to take into account the decrease in the total injected radioactivity due to voiding before imaging the patients in general.

The objectives of this work were first to experimentally measure the patient's body attenuation effect by performing direct dose rate measurements from patients undergoing PET/CT imaging studies using fluorodeoxyglucose ${ }^{18} \mathrm{~F}$ (FDG) and to compare the measurements with the reported air kerma rate constant proposed in the AAPM report, reported to be equal to $134 \mu \mathrm{Sv} / \mathrm{h} / \mathrm{GBq}$. The second objective was to quantify the effect of patients' bladder emptying on the measured dose rate values measured from the patients before and after voiding; and the last objective was to examine the effect of patients' body sizes metrics on the measured dose rates by performing statistical analysis using linear correlation methods. The aim of finding a correlation between the body size metrics and the measured dose rate per unit activity is to be able to predict with enough accuracy the dose rates around the patients in situations where actual dose rate measurements are not possible. Incident reconstruction scenarios are examples of situations, are accurate dose predictions and will help in putting the risk from radiation exposure in proper perspective.

\section{Materials and Methods}

\subsection{Measured dose Rate per Unit Activity}

The measured dose rate at voiding time divided by the activity calculated at the 
voiding time and corrected for radioactive decay was used for each patient to calculate the dose rate per unit activity constant and to compare it with the AAPM TG-108 proposed patient dose rate constant $(G)$ of $92 \mu \mathrm{Sv} \cdot \mathrm{m}^{2} / \mathrm{h} / \mathrm{GBq}$. the dose rate from the patient is given by the following equation:

$$
D_{P} / A=G C_{d} C_{P A}
$$

where $C_{d}$ is the distance correction factor or the inverse square law correction factor depending on the radiation source model, point or line source. In this work, we have measured the dose rate at $1 \mathrm{~m}$ and $C_{d}$ was assumed to be equal to 1 ;

$C_{P A}:$ is the patient attenuation correction factor;

$D_{P} / A$ : is the measured dose rate per unit activity.

Radiation dose measurements were done immediately before and after voiding, in order to calculate the dose rate reduction factor using equation 4 below. We have excluded from this analysis the patients with measured dose rate after voiding that were slightly higher than before voiding due to some mild urine contamination on their cloth. The total number of patients measurements reported in this study is 57, details are in Table 1.

The radiation dose rate was measured using a calibrated ionization chamber (SmartIon Type: 2120G; thermo Franklin, Massachusetts, USA). The FDG dose was administered using an automatic dose injector (Intego, by MedRad Inc, Indianola, PA, USA).

\subsection{Correction for Uptake Time}

The activity measured at the uptake time was calculated using the following relation:

Table 1. Patients data used in this study.

\begin{tabular}{cc} 
Parameter & Average \pm SD (min-max) \\
\hline Total number of patients & 27 \\
Male & 30 \\
Female & $22-79$ \\
Age range in [years] & $77 \pm 26(53-171)$ \\
Weight (W) in [kg] & $17 \pm 3(9-11)$ \\
Equivalent cylindrical diameter (ECD) in $[\mathrm{cm}]$ & $25 \pm 3(15-33)$ \\
Equivalent spherical diameter (ESD) in $[\mathrm{cm}]$ & $29 \pm 8(11-58)$ \\
Body mass index (BMI) & $47 \pm 14(13-99)$ \\
Ratio of Weight/Height $(\mathrm{W} / \mathrm{H})$ in $[\mathrm{kg} / \mathrm{m}]$ & $39 \pm 8(17-68)$ \\
Uptake time in [minutes] & $0.89 \pm 0.06(0.70-0.98)$ \\
patient voiding factor & $11 \% \pm 6 \%(2 \%-30 \%)$ \\
Voiding $\%$ & $92.2 \pm 14(65-136)$
\end{tabular}




$$
A\left(t_{u p}\right)=A_{0} \mathrm{e}^{-\lambda t_{u p}}
$$

where $t_{u p}$ : is the uptake time in minutes, and $\lambda$ : is equal to $\ln (2) / T_{1 / 2}$.

$T_{1 / 2}$ is the half-life of ${ }^{18} \mathrm{~F}=110$ minutes.

\subsection{Dose Rate Reduction due to Voiding}

We have calculated the patient voiding factor $(R)$ as the ratio of the dose rate measured after voiding over the dose rate measured before voiding:

$$
R=D_{\text {after }} / D_{\text {before }}
$$

The percentage of dose reduction due to voiding is then given by:

$$
D_{\text {reduction }}(\%)=(1-R) * 100 \%
$$

\subsection{Patient Body Density}

We have used a patient body density value of $1 \mathrm{~g} / \mathrm{cm}^{3}$ same to that of water as an acceptable assumption in this study.

\subsection{Equivalent Cylindrical Diameter (ECD)}

The volume $(V)$ of a cylinder with radius $(r)$ and simulating the patient with height $(H)$ is given by:

$$
V\left[\mathrm{~cm}^{3}\right]=2 \pi r^{2} H
$$

$$
V\left[\mathrm{~cm}^{3}\right]=M / \rho, \rho \text { in }\left[\mathrm{g} \cdot \mathrm{cm}^{-3}\right], M \text { is the mass in }[\mathrm{g}] \text { and } H \text { in }[\mathrm{cm}] .
$$

By Combining (4) and (5), we can calculate the cylindrical radius to be equal to:

$$
r=[(M / \rho) / 2 \pi H]^{1 / 2}
$$

where, $\rho$ is the density of water and equal to $1\left[\mathrm{~g} \cdot \mathrm{cm}^{-3}\right]$.

\subsection{Equivalent Spherical Radius (ESR)}

The volume of a sphere made of water and simulating a patient is given by:

$$
\begin{gathered}
V=4 / 3 \pi r^{3} \\
V\left[\mathrm{~cm}^{3}\right]=M / \rho, \rho \text { in }\left[\mathrm{g} \cdot \mathrm{cm}^{-3}\right] \text { and } M \text { in }[\mathrm{g}] \\
r^{3}=3 M / 4 \pi \rho, r=(3 M / 4 \pi \rho)^{1 / 3}
\end{gathered}
$$

With $\rho$ equal to the density of water: $1\left[\mathrm{~g} \cdot \mathrm{cm}^{-3}\right]$; the spherical radius is then will be equal to:

$$
r=(3 M / 4 \pi)^{1 / 3}
$$

This work was approved by the hospital medical research ethics committee.

\subsection{Statistical Analysis}

Pearson's $r$ correlation coefficient between the patient physical parameters and the dose rate measured were calculated using MATLAB Statistical Toolbox ver. 7.12. $r, r^{2}$ and $p$ values are given for each physical parameter assessed. 
The most significant parameter that may affect the dose rate was identified as the body mass index (BMI). All other tested parameters had statistical significant value above $99 \%, p$ values was less than 0.001 for all of the calculated patient's physical parameters assessed.

\section{Results}

\subsection{Patient self-Attenuation Factor}

We have measured the dose rate at one meter from the patient entrance body surface in the anterior direction. We have divided the measured dose rate per the injected activity to obtain a constant value of 92.2 with a standard deviation of 14 , a maximum value of 136 and a minimum of $65(\mu \mathrm{Sv} / \mathrm{h} / \mathrm{GBq})$; details are in Table 1. Our results are in close agreement with the recommended value of 92 $(\mu \mathrm{Sv} / \mathrm{h} / \mathrm{GBq})$ by the AAPM, TG-108 report.

\subsection{Effect of Voiding on the Measured dose Rate}

We have calculated the ratio of the dose rates measured after voiding to before voiding in order to present the results as percentage of the released activity due to voiding and to compare our measurements to the published data regarding the percentage of activity excreted by the patient due to voiding. The amount of voided activity will contribute to the dose reduction in the bladder of the patient (Hays et al., 1998) [2].

We have quantified the effect of voiding on the measured dose rate from the patients and found it to reduce the dose rate by $11 \%$. The literature reports reduction in the order of $15 \%$ of the injected activity for the first 2 hours post injection of the FDG [3]. We have found a wide variation for the first void time, our average time measured before the first void was $39 \pm 8$ minutes; other studies reports different values; $83 \pm 19$ minutes and $77 \pm 18$ minutes [4] [5] [6].

It is also worth to mention that we have excluded certain number of patient data from our study because the measured dose rate after voiding was higher than the one measured before. We concluded that these patients had accidentally contaminated their cloth with radioactive urine during voiding, which caused the dose rate after voiding to be higher.

\subsection{Correlation of Patient's Body Metrics and the Measured dose Rate}

We have used five metrics to represent the patient size, the patient body weight $(\mathrm{W})$ in $[\mathrm{kg}]$, the body mass index (BMI) in $\left[\mathrm{kg} / \mathrm{m}^{2}\right]$, the ratio of patient weight to height $(\mathrm{W} / \mathrm{H})$ in $[\mathrm{kg} / \mathrm{m}]$, the equivalent cylindrical diameter $(\mathrm{ECD})$ in $[\mathrm{cm}]$, which is a cylinder full of water and having the same weight and height as the patient given by (Equation (6)) and the equivalent spherical radius (ESR) in $[\mathrm{cm}]$,which is a sphere made of water having the same mass as the patient given by (Equation (9)).

We have measured the strength of the association between the dose rates per 
unit activity and the patient body size metrics using statistical parameters: the coefficient of correlation ( $r$ ), the coefficient of determination $\left(r^{2}\right)$ and the significance level $(\mathrm{p})$ value. MATLAB statistics toolbox ver. 7.12.0 was used to perform the statistical analysis.

For all of the 57 patients, the measured dose rate per unit activity in $[\mu \mathrm{Sv} / \mathrm{h} / \mathrm{GBq}]$ as a function of the patients' body size metrics were plotted as shown in (Figures 1-5).

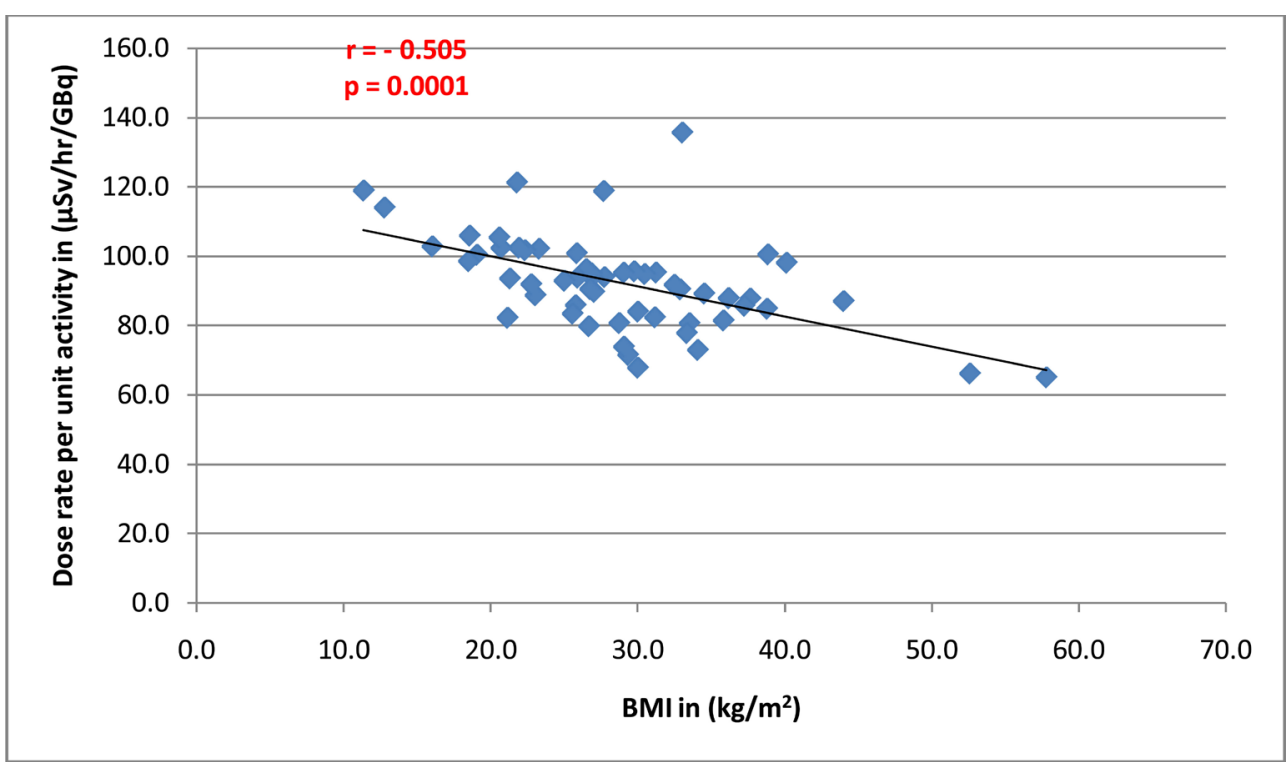

Figure 1. Graph of the measured dose rate per unit activity in $(\mu \mathrm{Sv} / \mathrm{h} / \mathrm{GBq})$ as a function of the patient body mass index (BMI). We notice the negative slope of the trend line indicating that when the patient BMI increases the dose rate per unit activity decrease due to attenuation of the radiation by the patient body.

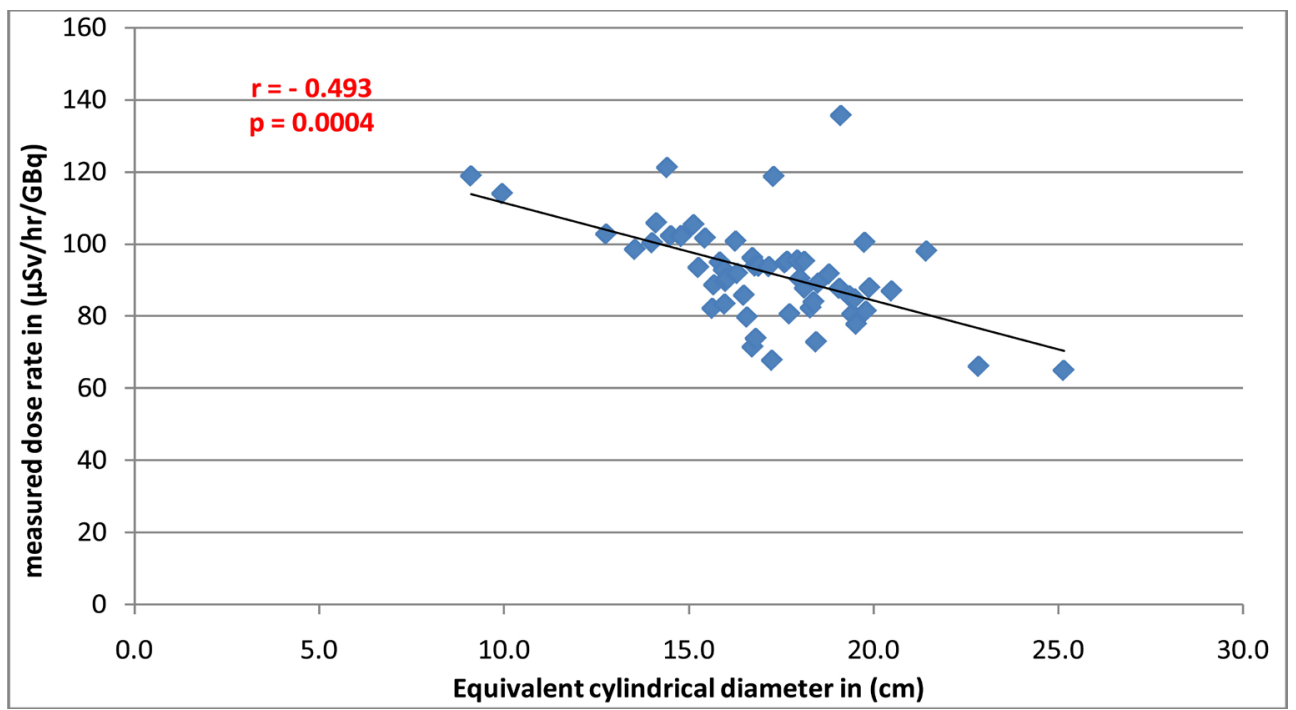

Figure 2. Graph of the measured dose rate per unit activity in $(\mu \mathrm{Sv} / \mathrm{h} / \mathrm{GBq})$ as a function of the patient equivalent cylindrical diameter (ECD). We notice the negative slope of the trend line indicating that when the patient ECD increases the dose rate per unit activity decrease due to attenuation of the radiation by the patient body. 


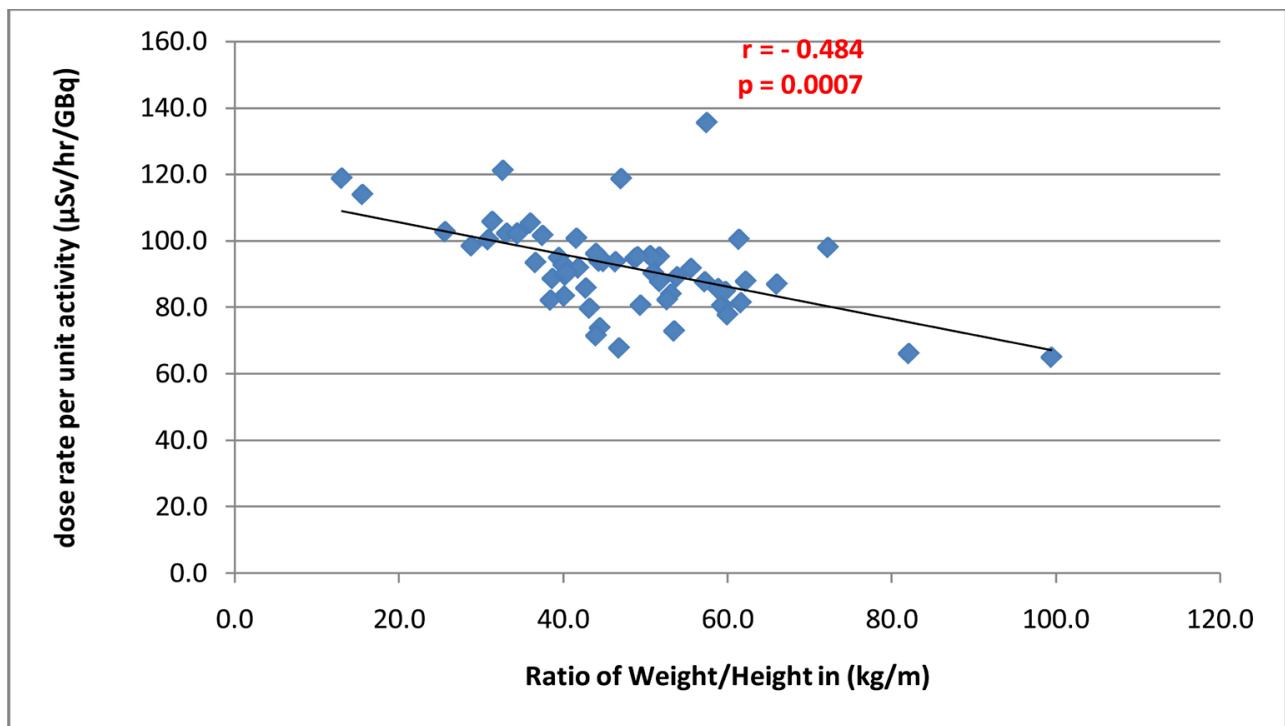

Figure 3. Graph of the measured dose rate per unit activity in $(\mu \mathrm{Sv} / \mathrm{h} / \mathrm{GBq})$ as function of the ratio of the patient weight to the patient height $(\mathrm{W} / \mathrm{H})$. We notice the negative slope of the trend line indicating that when $\mathrm{W} / \mathrm{H}$ increases the dose rate per unit activity decrease due to attenuation of the radiation by the patient body.

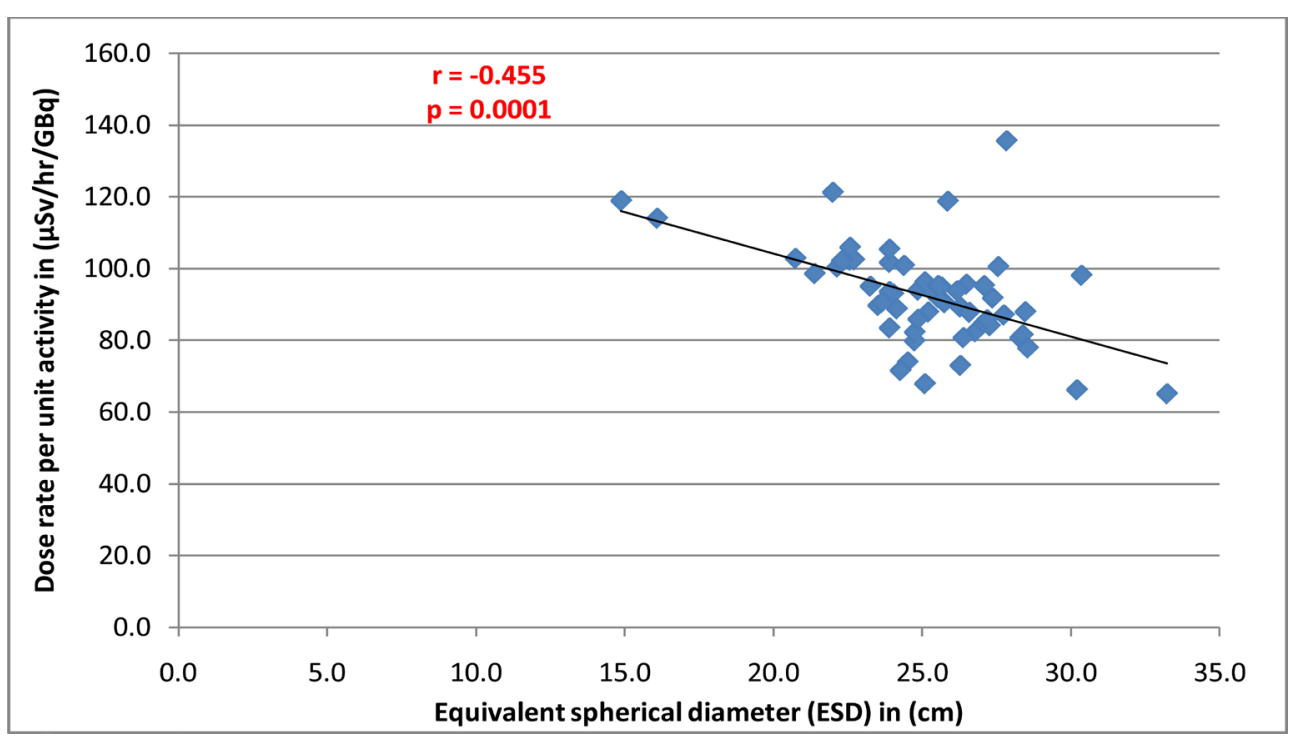

Figure 4. Graph of the measured dose rate per unit activity in $(\mu \mathrm{Sv} / \mathrm{h} / \mathrm{GBq})$ as a function of the patient equivalent spherical diameter (ESD). We notice the negative slope of the trend line indicating that when the patient ESD increases the dose rate per unit activity decrease due to attenuation of the radiation by the patient body.

We have obtained a weak correlation $(r<0.51, p<0.001)$ between the measured dose rate constant and the patient body size parameters $(\mathrm{BMI}, \mathrm{ECD}, \mathrm{W} / \mathrm{H}$, ESD and W). With the BMI having the strongest correlation coefficient at ( $r=$ $-0.505, p=0.0001$ ). The negative correlation coefficients means when the patient size metrics increase the dose rate from the patient decrease which is normally due to the patient body attenuation factor that reduces the measured dose rate form the radiation sources distributed inside the patient body organs (Table 2). 


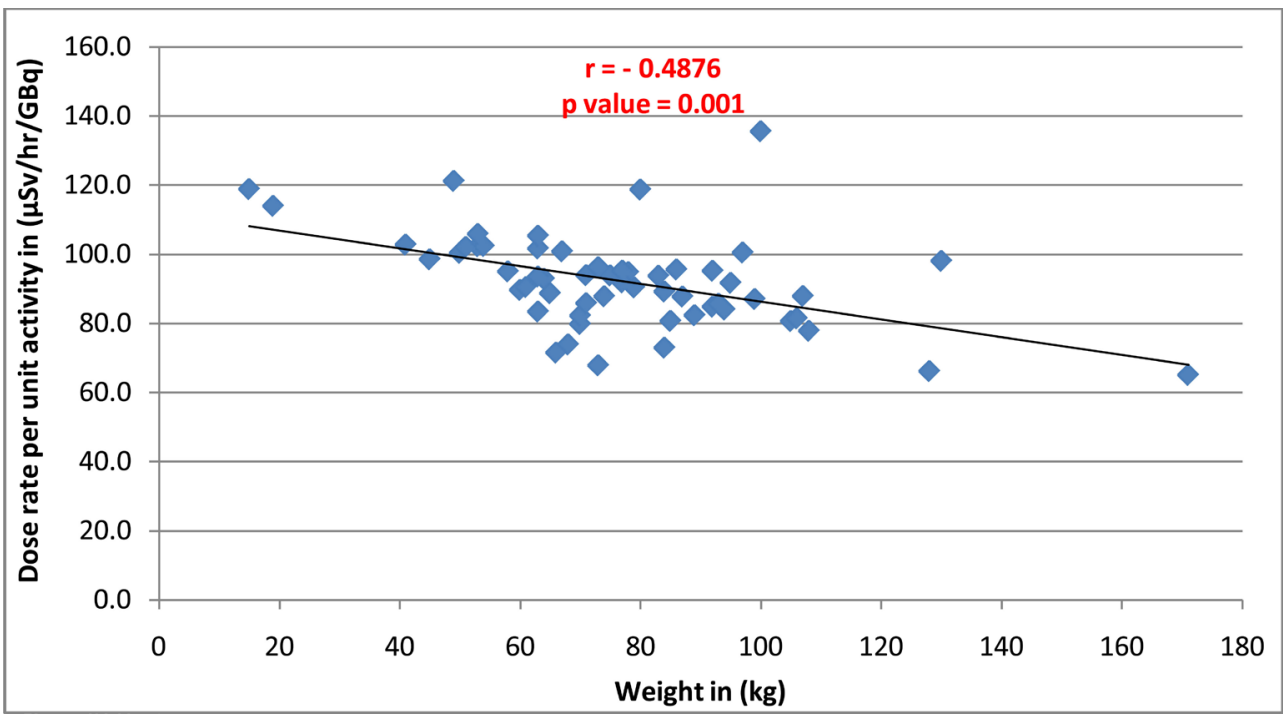

Figure 5. Graph of the measured dose rate per unit activity in $(\mu \mathrm{Sv} / \mathrm{h} / \mathrm{GBq})$ as a function of the patient weight $(\mathrm{W})$. We notice the negative slope of the trend line indicating that when the patient weight increases the dose rate per unit activity decrease due to attenuation of the radiation by the patient body.

Table 2. Correlation parameter between the patient physical data parameters and the dose rate measured from the patient at one meter. $r, r^{2}$ and $p$ values are given for each parameter in decreasing order.

\begin{tabular}{cccc}
\hline Parameter & $\mathbf{r}$ & $\mathbf{r}^{2}$ & $\mathbf{p}$ \\
\hline Body Mass Index [BMI] & -0.505 & 0.255 & 0.0001 \\
Equivalent Cylindrical Diameter [ECD] & -0.493 & 0.243 & 0.0004 \\
Weight/Height ratio [W/H] & -0.484 & 0.235 & 0.0007 \\
Equivalent Spherical Diameter [ESD] & -0.455 & 0.207 & 0.0001 \\
Weight [W] & -0.442 & 0.195 & 0.0002 \\
\hline
\end{tabular}

The most significant parameter that may affect the dose rate is the body mass index (BMI). All tested parameters had statistical significant value above $99 \%$, p values was less than 0.001 .

Because of the strong heterogeneity of the radioactive material (the FDG) distribution inside the patient body it was very difficult to predict the external radiation dose rate measured at one meter from the patient by using only the patient body size metrics and the injected activity. Therefore we conclude that it is not possible to accurately estimate the radiation dose rate at one meter from the patient without performing actual radiation dose measurements.

\section{Discussion}

As patient weight increases, fewer photons are getting out of the body [7]. High photon attenuation and scatter in obese patients affect image quality [8] [9]. Masuda et al., 2009 [10] demonstrated that the quality of 18F-FDG PET/CT images of overweight patients is often degraded. This fact is apparently due to the patient self-shielding effect. 
Self-attenuation of radiation by patient's bodies was quantified, and found to cause a significant decrease in radiation exposure of more than $40 \%$ due to non-uniform distribution of FDG and attenuation within the patients [11].

Quinn et al., 2012 [12] support the use of $0.092 \mu \mathrm{Sv} / \mathrm{hr} / \mathrm{MBq}$ measured at 1 meter from the chest of patients immediately following injection or 0.067 at 60 min post injection as reasonable representation of the dose rate.

Yi et al., 2013 [13] have compared the measured and calculated dose rates from the radioactive patient and found that the calculated values were always higher than measured values and suggested the application of self-shielding factors.

In most cases the patient will void prior to imaging, removing approximately $15 \%-20 \%$ of the administered activity and thereby decreasing the dose rate by 0.85 [1]. The initial voiding time seems to play a role in the dose calculations to the bladder wall; the optimum initial voiding time to deliver the lowest dose according to the traditional MIRD static bladder model is 40 minutes [14].

PET image quality depends on patient weight and habitus; decreasing image quality is associated with an increasing weight and body mass index [15]. It was proposed that ${ }^{18} \mathrm{~F}$-FDG dose injected should be adjusted to both body weight and height [6]. Cylindrical phantoms have been used to simulate patients with different body masses in order to optimize the FDG dose regime for imaging studies [16].

\section{Conclusions}

The measured dose rate per unit on injected activity in this study was in agreement with the recommended value by the AAPM TG-108. Patient bladder voiding before scanning reduced the measured dose rate at one meter from the patient by about $11 \%$. Finally, patient body size metrics cannot be used solely to predict the dose rate levels expected from individual patient without performing actual radiation dose rate measurements.

The activity normalized dose rate constant of $92(\mu \mathrm{Sv} / \mathrm{h} / \mathrm{GBq})$ measured at one meter anterior to the patient can be used with confidence to estimate dose rates from patients undergoing PET/CT imaging using FDG, because it includes patient body attenuation and scatter factor.

The presented information will benefit medical physicist working in nuclear medicine, radiation safety policy makers and regulators.

\section{Conflicts of Interest}

The authors declare no conflicts of interest regarding the publication of this paper.

\section{References}

[1] Madsen, M.T., Anderson, J.A., Halama, J.R., Kleck, J., Simpkin, D.J., Votaw, J.R., Wendt III, R.E., Williams, L.E. and Yester, M.V. (2006) AAPM Task Group 108: PET and PET/CT Shielding Requirements. Medical Physics, 33, 4-15. https://doi.org/10.1118/1.2135911 
[2] Hays, M.T. and Segall, G.M. (1998) A Mathematical Model for the Distribution of Fluorodeoxyglucose in Humans. Journal of Nuclear Medicine, 40, 1358-1366.

[3] Jones, S.C., Alavi, A., Christman, D., Montanez, I., Wolf, A.P. and Reivich, M. (1982) The Radiation Dosimetry of 2-[F-18]Fluoro-2-Deoxy-D-Glucose in Man. Journal of Nuclear Medicine, 23, 613-617.

[4] Mejia, A.A., Nakamura, T., Masatoshi, I., Hatazawa, J., Masaki, M. and Shoichi, W. (1991) Estimation of Absorbed Doses in Humans due to Intravenous Administration of Fluorine-18-Fluorodeoxyglucose in PET Studies. Journal of Nuclear Medicine, 32, 699-706.

[5] Dowd, M.T., Chen, C.T., Wendel, M.J., Faulhaber, P.J. and Cooper, M.D. (1991) Radiation Dose to the Bladder Wall from 2- $\left[{ }^{18} \mathrm{~F}\right]$ Fluoro-2-Deoxy-D-Glucose in Adult Humans. Journal of Nuclear Medicine, 32, 707-712.

[6] Cho, I.H., Han, E.O. and Kim, S.T. (2014) Very Different External Radiation Doses in Patients Undergoing PET/CT or PET/MRI Scans and Factors Affecting Them. Hellenic Journal of Nuclear Medicine, 17, 13-18.

[7] Watson, C.C., Casey, M.E., Bendriem, B., Carney, J.P., Townsend, D.W., Eberl, S., Meikle, S. and Di Filippo, F.P. (2005) Optimizing Injected Dose in Clinical PET by Accurately Modeling the Counting-Rate Response Functions Specific to Individual Patient Scans. Journal of Nuclear Medicine, 46, 1825-1834.

[8] Halpern, B.S., Dahlbom, M., Auerbach, M.A., Schiepers, C., Fueger, B.J., Weber, W.A., Silverman, D.H.S., Ratib, O. and Czernin, J. (2005) Optimizing Imaging Protocols for Overweight and Obese Patients: A Lutetium Orthosilicate PET/CT Study. Journal of Nuclear Medicine, 46, 603-607.

[9] Halpern, B.S., Dahlbom, M., Quon, A., Schiepers, C., Waldherr, C., Silverman, D.H., Ratib, O. and Czernin, J. (2004) Impact of Patient Weight and Emission Scan Duration on PET/CT Image Quality and Lesion Detectability. Journal of Nuclear Medicine, 45, 797-801.

[10] Masuda, Y., Kondo, C., Matsuo, Y., Uetani, M. and Kusakabe, K. (2009) Comparison of Imaging Protocols for 18F-FDG PET/CT in Overweight Patients: Optimizing Scan Duration Versus Administered Dose. Journal of Nuclear Medicine, 50, 844-848. https://doi.org/10.2967/jnumed.108.060590

[11] Zeff, B.W. and Yester, M.V. (2005) Patient Self-Attenuation and Technologist Dose in Positron Emission Tomography. Medical Physics, 32, 861-865. https://doi.org/10.1118/1.1869552

[12] Quinn, B., Holahan, B., Aime, J., Humm, J., St-Germain, J. and Dauer, L.T. (2012) Measured Dose Rate Constant from Oncology Patients Administered 18F for Positron Emission Tomography. Medical Physics, 39, 1071-1079. https://doi.org/10.1118/1.4749966

[13] Yi, Y., Stabin, M.G., McKaskle, M.H., Shone, M.D. and Johnson, A.B. (2013) Comparison of Measured and Calculated Dose Rates Near Nuclear Medicine Patients. Health Physics, 105, 187-191. https://doi.org/10.1097/HP.0b013e318290cc0e

[14] Wu, T.H., Liu, R.S., Dong, S.L., Chung, Y.W., Chou, K.L. and Lee, J.S. (2002) Dynamic Evaluation of Absorbed Dose to the Bladder Wall with a Ballon-Bladder Phantom during a Study Using 18-F Fluorodeoxyglucose Positron Emission Imaging. Nuclear Medicine Communications, 23, 749-755. https://doi.org/10.1097/00006231-200208000-00008

[15] Accorsi, R., Karp, J.S. and Surti, S. (2010) Improved Dose Regimen in Pediatric PET. Journal of Nuclear Medicine, 51, 293-300.

https://doi.org/10.2967/jnumed.109.066332 
[16] De Groot, E.H., Post, N., Boellaard, R., Wagenaar, N.R.L., Willemsen, A.T.M. and Van Dalen, J.A. (2013) Optimized Dose Regimen for Whole-Body FDG-PET Imaging. EJNMMI Research, 63, 1-11. https://doi.org/10.1186/2191-219X-3-63 